\title{
$1 \quad$ Vessel Route Choice Theory and Modeling
}

2 Yaqing Shu, PhD candidate

Department of Transport \& Planning

Faculty of Civil Engineering and Geosciences

Delft University of Technology

Stevinweg 1, PO Box 5048, 2600 GA Delft - The Netherlands

Phone +31 152787690

Fax +31 152783179

e-maily.shu@tudelft.nl

Winnie Daamen, Associate Professor

Department of Transport \& Planning

Faculty of Civil Engineering and Geosciences

Delft University of Technology

Stevinweg 1, PO Box 5048, 2600 GA Delft - The Netherlands

Phone +31 152785927

Fax +31 $\quad+32783179$

e-mail w.daamen@tudelft.nl

Han Ligteringen, Professor

Department of Hydraulic Engineering

Faculty of Civil Engineering and Geosciences

Delft University of Technology

Stevinweg 1, PO Box 5048, 2600 GA Delft - The Netherlands

Phone +31 152784285

Fax +31152783179

e-mail h.ligteringen@tudelft.nl

Serge Hoogendoorn, Professor

Department of Transport \& Planning

Faculty of Civil Engineering and Geosciences

Delft University of Technology

Stevinweg 1, PO Box 5048, 2600 GA Delft - The Netherlands

Phone +31 152785475

Fax +31152783179

e-mail s.p.hoogendoorn@tudelft.nl

\begin{tabular}{|l|r|}
\hline Word count \\
\hline Abstract & 236 \\
Main text \& Reference & 5015 \\
Figures $(7 \times 250)$ & 1750 \\
Tables $(1 \times 250)$ & 250 \\
\hline Total & $\mathbf{7 2 5 1}$ \\
\hline
\end{tabular}


Shu, Daamen, Ligteringen and Hoogendoorn

\section{ABSTRACT}

To better describe vessel traffic in ports and inland waterways, a new maritime traffic model is developed. In our research, vessel behavior is categorized into a tactical level (route choice) and an operational level (the dynamics of the vessel behavior). This new maritime traffic model comprises two parts: the route choice model resulting in the vessel's preferred route, and the operational model describing the maneuvering behavior including interactions between vessels.

This paper presents the vessel route choice model, which is based on disutility or cost minimization. The cost is determined by characteristics of the infrastructure, such as expected sailing time and distance to the bank. It is assumed that the bridge team will try to follow a preferred route that minimizes the cost to the destination. To calculate this preferred route to the certain destination, the so-called value function is defined as the minimum disutility function in continuous time and space. Subsequently, the value function is solved using dynamic programming and numerical solution approach.

Based on an Automatic Identification System (AIS) dataset of unhindered vessel behavior in the Port of Rotterdam, the vessel route choice model is calibrated. The calibrated results of the route choice model show plausible preferred routes in the research area, which help us to understand the desired vessel behavior (route). These results could be used to improve vessel traffic management and provide a basis for predicting vessel behavior at the operational level. 


\section{INTRODUCTION}

Due to the globalization of products trade, the usage of vessels for transportation increases all over the world. It is getting more and more important to find the balance between safety and capacity in busy ports and inland waterways: when measures are taken to increase capacity, usually the safety decreases. To optimize ports and waterway design and improve maritime traffic management, modeling tools can be used.

Vessel behavior including its speed and path is difficult to predict, especially in ports and inland waterways, since a lot of factors influence vessel behavior, such as waterway's geometry, human factors and external conditions including wind and visibility. Currently, some maritime models focus on calculating the risk probability of collisions or groundings (1-3), while other models mainly consider the hydrodynamics of vessels (4-6) or simulate the routing in a shipping network (7-9). In addition, most maritime simulation models focus on vessel dynamic and traffic for open seas. These models cannot be applied in constrained ports and waterways due to the fact that different factors affect sailing behavior in ports and waterways than in open seas (e.g. influence of banks and influence of water depth). Little research has been performed regarding the vessel route choice in inland waterways, interaction between vessels and human factors influencing maritime traffic. In order to optimize ports and waterway design and improve maritime traffic management, a new model needs to be developed to describe vessel traffic in ports and inland waterways.

In our research, vessel behavior is categorized into a tactical level and an operational level (10). The tactical level includes vessel route choice in inland waterways without external influences. The vessel route choice at the tactical level serves as the basis for vessel behavior at the operational level. The operational level includes the external influences and dynamics of the vessel behavior, e.g. all decisions related to the sailing taken for the coming short time period. In other words, at the operational level, it is hypothesized that vessels follow the preferred route generated at the tactical level as much as possible, while taking into account external influences and human factors. Therefore, our new maritime traffic model will comprise two parts: the route choice model resulting in preferred routes, and the operational model describing the sailing behavior including interactions between vessels, which was proposed in our previous research (11). This paper presents the vessel route choice model at the tactical level.

In vessel route choice theory, it is assumed that disutility or cost of each route for the vessel determined by characteristics of the infrastructure, such as expected sailing time and distance to the bank. The bridge team will try to follow a route that minimizes the disutility to reach their destination, being the preferred route. To calculate the preferred route, the so-called value function is defined as the expected minimum disutility function in continuous time and space. From this value function, the preferred route can be derived from the present position to the destination, which leads to the least disutility to the vessel. In other words, the bridge team will navigate their vessel in the direction in which the cost decreases most rapidly. The value function is obtained using dynamic programming and a numerical solution approach.

In recent research, AIS data have been proven to be a powerful tool to investigate maritime traffic $(12,13)$. Automatic Identification System (AIS) is an onboard system transmitting vessel information (position, velocity, destination, etc.) between nearby vessels and shore stations. AIS data in the Port of Rotterdam are provided by the Maritime Research Institute Netherlands (MARIN, the leading institute for hydrodynamic research and maritime technology in the Netherlands). These data are used for model calibration in this paper.

The remainder of this paper is structured as follows. Firstly, the vessel behavior theory at the tactical level is proposed, followed by an optimal route choice model for vessels in ports and restricted waterways. Then, the calibration process and results of the vessel route choice model are described. Finally, conclusions and recommendations for future research are presented.

\section{VESSEL BEHAVIOR AT THE TACTICAL LEVEL}

Our research focuses on the vessel behavior in the two-dimensional space, including vessel position and velocity. Our previous research $(14,15)$ showed that a lot of factors influence vessel behavior, such as vessel characteristics (e.g. vessel type and size), waterway geometry and external conditions (e.g. wind, visibility and current).

As mentioned before, we investigate vessel route choice at the tactical level. In the approach, the bridge team is considered as the "brain" of the vessel. In the vessel route choice theory, it is assumed that disutility or cost of each route for the vessel is determined by characteristics of the infrastructure, which will be proposed and included in the running cost in the next section. To identify the preferred route, the bridge team will predict and minimize this expected disutility or $\operatorname{cost} C$.

In our research, we investigate vessel behavior in a waterway stretch, which is defined by two cross sections. These two cross sections can be considered as the entrance and the destination for vessels sailing in this direction. The vessel route $x(\cdot)$ is a continuous function, uniquely determined by the velocity trajectory $v(\cdot)$ through 
the waterway. Since the position is the derivative of the velocity, so optimizing the velocity also optimizes the route.. Then, the utility optimization for the vessel route will yield the optimized velocity choice at the tactical level.

It should be noted here that both vessel course and speed are included in this optimized velocity. As we discussed before, vessel speed is influenced by external influences (e.g. wind and visibility) and is determined by the bridge team according to the traffic situation and the infrastructure at the operational level. Therefore, the vessel route choice model will mainly consider vessel course, rather than vessel speed.

In equation (1), the optimal course (over a time period) is the defined as that minimizes the cost, given the current time and position of the vessel:

$$
v^{*}(\cdot)=\arg \min C\left(v(\cdot) \mid t_{0}, x_{0}\right)
$$

where $t_{0}$ and $x_{0}$ are the current time and position of the vessel. This way, the vessel route choice problem becomes the optimization for vessel velocity in the research area.

In the next section, we will discuss the expected disutility and the solution for the vessel route choice optimization.

\section{OPTIMAL ROUTE CHOICE FOR VESSELS}

With respect to the vessel behavior at the tactical level, it is assumed that the bridge team chooses a route by predicting and minimizing the expected disutility of following this route, which is determined by characteristics of the infrastructure. The contributions of these characteristics to the cost $C$ will be introduced in following sections.

The decision making process of the bridge team is feedback-oriented. That means for each time step, the bridge team will reconsider the expected disutility and make the choice for the preferred route in the next time steps to minimize the expected cost. This is a continuous feedback control system including input (velocity) and the controlled output (location).

As we know, vessels sometimes deviate from their planned path when they encounter other vessels. To flexibly adapt vessels to other routes, the expected minimum perceived disutility for all locations $x$ and instants $t$ is proposed. The so-called value function $W(t, x)$ is defined as expected minimum perceived disutility function in continuous time and space (16). Based on the solution of $W(t, x)$, the optimal route choice for vessels can be determined.

\section{Vessel kinematics under uncertainty}

As mentioned above, velocity and location are considered as control input and output, respectively. To apply the control, consider the location $x$ (the state) and the velocity $v$ (the control) for a vessel. The vessel position at instant $t$ $x(t)$ is known to the bridge team and expressed by $\hat{x}$. Then, the bridge team will predict the route costs and determine the future position $x(\tau)$ for $\tau>t$ using the vessel kinematics:

$$
\mathrm{d} x=v \mathrm{~d} t+\mathrm{d} \varepsilon \quad \text { subject to } x(t)=\hat{x}
$$

where $v=v(\tau)$ denotes velocity of the vessel for $\tau>t$. The term $\mathrm{d} \varepsilon$ represents the small disturbance, which is $N\left(0, \sigma^{2}\right)$-distributed. The white noise reflects the uncertainty in the expected traffic conditions and is caused by lack of experience or randomness of future conditions.

Here, we investigate vessels sailing in ports and waterways, where they sail at relatively low speed. This speed is around 10 knots, which is normally far below the physical limitation of the vessel. So this physical limitation is not considered in our research.

\section{Generalized expected utility}

In vessel route choice, we investigate vessel velocity and position in waterways. Consider a part of waterway between two cross sections, which are set as the origin and the destination respectively.

Let $\left[t, t_{t}\right)$ denote the planning period of the bridge team, where $t$ and $t_{t}$ are respectively the current time and the terminal time (planning horizon). The vessel is expected to reach its destination during this time period. Let $t_{a}$ denote the time of arrival at the destination, and let $T=\min \left(t_{t}, t_{a}\right)$. Consider an arbitrary control $v_{[t, T)}$ resulting in the trajectory $x_{[t, T)}$, the expected disutility or cost $C$ is defined as

$$
C\left(T, v_{[t, T)}\right)=\int_{t}^{T} L(\tau, x(\tau), v(\tau)) \mathrm{d} \tau+\phi(T, x(T))
$$

where $L$ and $\phi$ respectively denote the so-called running cost and the terminal cost. The running cost $L(\tau, x(\tau), v(\tau))$ reflects the cost incurred in a small time period $[\tau, \tau+\mathrm{d} \tau)$, given the location $x(\tau)$ at time $\tau$ and 
control velocity $v(\tau)$. The terminal cost $\phi(T, x(T))$ reflects the penalty incurred due to the vessel ending up at position $x(T)$ at the terminal time $T$, but not at the destination. This expected utility is input into the dynamic programming problem identified later.

\section{Specification of terminal cost}

As defined in the previous section, the terminal time $T$ either equals the final time $t_{t}$ of the planning period or the time $t_{a}$ at which the vessel arrives at the destination. The terminal cost is defined as

$$
\phi(T, x(T))= \begin{cases}0, & T<t_{t} \\ \phi, & T=t_{t}\end{cases}
$$

The terminal cost $\phi$ thus reflects the penalty for not having arrived at the destination at the end of the prediction horizon. When the vessel arrives at the destination in time, the penalty is zero. So the vessel will aim to reach the destination within the prediction horizon.

\section{Specification of running cost}

By definition, the running cost $L$ reflects the influence of different characteristics of the infrastructure considered by the bridge team. For simplicity, it is assumed that these attributes are independent and the running cost is linear-inparameters as follows:

$$
L(t, x, v)=\sum_{k=1,2, \ldots} c_{k} L_{k}(t, x, v)
$$

where $L_{k}$ denote the contributions on vessel route choice of $k$ different characteristics of the infrastructure, and $c_{k}$ are relative weights for these factors. It should be noted that all weights cannot be uniquely determined from AIS data, since only the relative importance of the weights can be found. Furthermore, weight factors $c_{k}$ are different for different vessel groups according to AIS data analysis. For example, small vessels follow a path closer to their starboard bank compared to large vessels.

The data analysis showed that both banks and the vessel characteristics have influence on vessel route choice (14). In our approach, we consider the following characteristics of the infrastructure in the running costs for a specific vessel category: expected sailing time, counteract the bend waterway effect explained below, discomfort due to proximity to banks and sailing at a certain speed. These running costs are described below.

\section{Expected sailing time}

For the expected sailing time, we define $L_{1}$ as follows:

$$
L_{1}(t, x, v)=1
$$

The definition above results in the route cost

$$
\int_{t}^{T} c_{1} \cdot L_{1}(\tau, x(\tau), v(\tau)) d \tau=\int_{t}^{T} c_{1} d \tau=c_{1}(T-t)
$$

It means that the contribution of expected sailing time on running cost equals the expected sailing time, multiplied by the weight $c_{1}$. The weight factor $c_{1}$ reflects the time-pressure for the bridge team to arrive in time at their destination.

\section{The waterway bend effect}

By including the sailing time, as stated in the previous paragraph, we assume that vessels prefer to sail in a straight line towards their destination. In bended waterways, this implies that vessels will cut corners. However, previous AIS data analysis (14) showed that vessels normally sail along the centerline of the waterway in the bend area of the waterway. In the route choice model, a term is therefore added to the running cost to counteract the bend waterway effect to make sure vessels are sailing along the waterway in the bend area. An example of a bend waterway is shown in FIGURE 1, where the bend area is shadowed. In the figure, $\theta$ denotes the change of waterway direction before the bend and after the bend. $S$ denotes the average arc length, which approximately equals the length of the middle line of the bend waterway. Then, $\theta / S$ reflects the direction change in unit distance, which could be defined as strength of the bend. $d_{c v}$ is the distance to the convex bank.

To counteract this influence caused by a bend in waterways, a linear decreasing utility from the convex bank is defined as $L_{2}$ as follows 


$$
L_{2}=-\frac{\theta}{S} \cdot d_{c v}(x)
$$

It should be noted that this cost is only added in the bend area of the waterway. Then, in the bend area, $L_{2}$ provides repulsion from the convex bank.

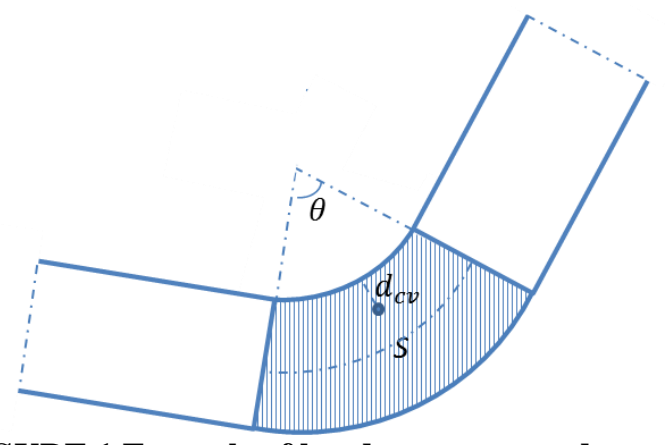

FIGURE 1 Example of bend waterway and parameters.

Discomfort due to proximity to banks

As we know from AIS data analysis, sailing vessels normally keep a certain distance to the bank, which in the present case has been defined as the five meter water depth line. The bridge team will adjust its course to make sure that their vessel is not too close to either portside bank or starboard bank. In our approach, it is assumed that a vessel is influenced by the bank when it is closer to the bank than a certain threshold distance. As shown in FIGURE 2, a vessel sails to the right and its present location is $x$. Let $d_{1}(x)$ and $d_{2}(x)$ denote the distance to the portside bank and the starboard bank respectively, $R_{1}$ and $R_{2}$ describe how far both banks can influence the vessel. The vessel is influenced by the portside bank only when it sails in Area 1, which means that $d_{1}(x)$ is less than $R_{l}$. The starboard bank influences the vessel in a similar way. In Area 3, the vessel is not influenced by either bank.

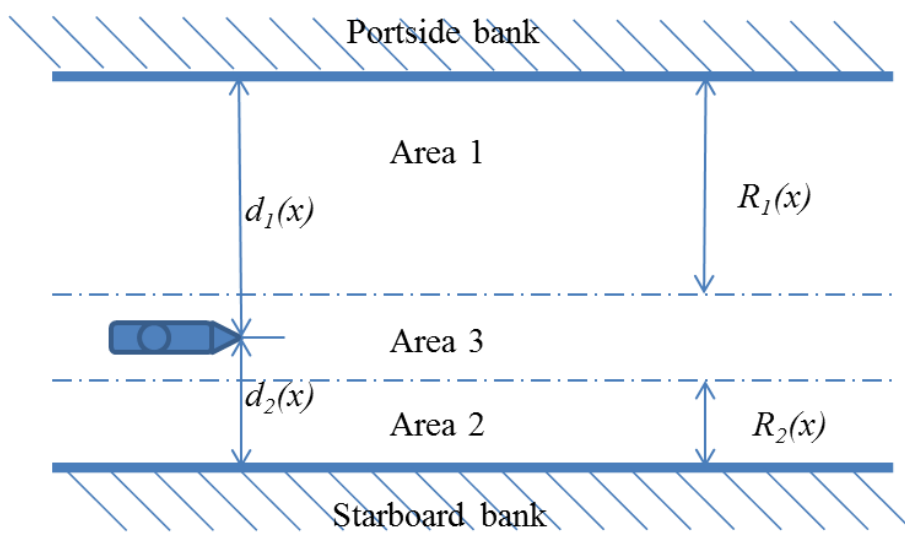

\section{FIGURE 2 Waterway area division according to portside and starboard bank.}

We add the influence of the two banks in the expected route cost as a monotonously decreasing (linear) function of the distance to the bank in the corresponding area. Running cost components $L_{3}$ and $L_{4}$ denote the contributions from the portside and starboard bank, respectively. They are defined as

$$
\begin{aligned}
& L_{3}(t, x, v)=\left\{\begin{array}{rr}
0, & d_{1}(x)>R_{1} \\
\frac{R_{1}-d_{1}(x)}{R_{1}}, & d_{1}(x) \leq R_{1}
\end{array}\right. \\
& L_{4}(t, x, v)=\left\{\begin{array}{rr}
0, & d_{2}(x)>R_{2} \\
\frac{R_{2}-d_{2}(x)}{R_{2}}, & d_{2}(x) \leq R_{2}
\end{array}\right.
\end{aligned}
$$




$$
\begin{aligned}
& R_{1}=\left[d_{1}(x)+d_{2}(x)\right] \cdot r_{1} \\
& R_{2}=\left[d_{1}(x)+d_{2}(x)\right] \cdot r_{2}
\end{aligned}
$$

where $r_{1}$ and $r_{2}$ describe the percentage of the waterway width, in which both banks influence vessel behavior and contribute to the cost function.

\section{Sailing at a certain speed}

To arrive at the destination in time, it is necessary to have an appropriate speed. However, high speed means high energy consumption, which will result in high cost. Speed choice is thus a trade-off between the time remaining to sail to the destination and the energy consumption due to sailing at a certain speed. For simplicity we assume the energy consumption to be a quadratic function of the vessel speed as follows:

$$
L_{5}(t, x, v)=\frac{1}{2} v^{2}
$$

\section{Modeling by dynamic programming and numerical solution}

To solve the route choice problem in continuous time and space, the so-called value function $W(t, x)$ is defined as the expected minimum perceived disutility function. To solve the value function, a dynamic programming approach and a numerical solution approach are used in the model. The solution of $W(t, x)$ describes the minimum cost to the destination for a vessel located at position $x$ at instant $t$. Based on this solution, the optimal course and speed can be determined. For details, we refer to previous work (17).

\section{ROUTE CHOICE MODEL CALIBRATION}

In this section, the vessel route choice model is calibrated using AIS data. We firstly introduce the AIS data and unhindered vessel behavior, being the vessel behavior without influence of other vessels. As we discussed before, vessel encounters are considered at the operational level, but not at the tactical level. So only unhindered vessel behavior should be used to calibrate the vessel route choice model at the tactical level. Then, the calibration set-up and the objective function for calibration are described. Finally, calibration results are presented.

\section{AIS data and unhindered paths}

In our research, the class of small General Dry Cargo (GDC) vessels less than 3600 gross tonnage is used. AIS data of these vessels in the Botlek area in the Port of Rotterdam from January 2009 to April 2011 are selected. As shown in FIGURE 3, the research area is called "Sea-Nieuwe Maas", which is corresponding to vessels sailing from the Sea (in the west) to the Nieuwe Maas river (in the east). To compare lateral positions of these tracks and easily calculate the average path, 69 cross sections with intervals around 50 meters are defined in the research area. These cross sections are approximately perpendicular to the waterway axis and used to select AIS data. Endpoints of these cross sections are located at the five meters water depth line, since it is found in the data analysis that vessels will not pass the five meters depth line. For the areas without five meters water depth line, such as entrances to basins or waterway branches, endpoints are created such that the boundary remains smooth. In the model, these five meters water depth lines will form the effective waterway for vessel sailing.

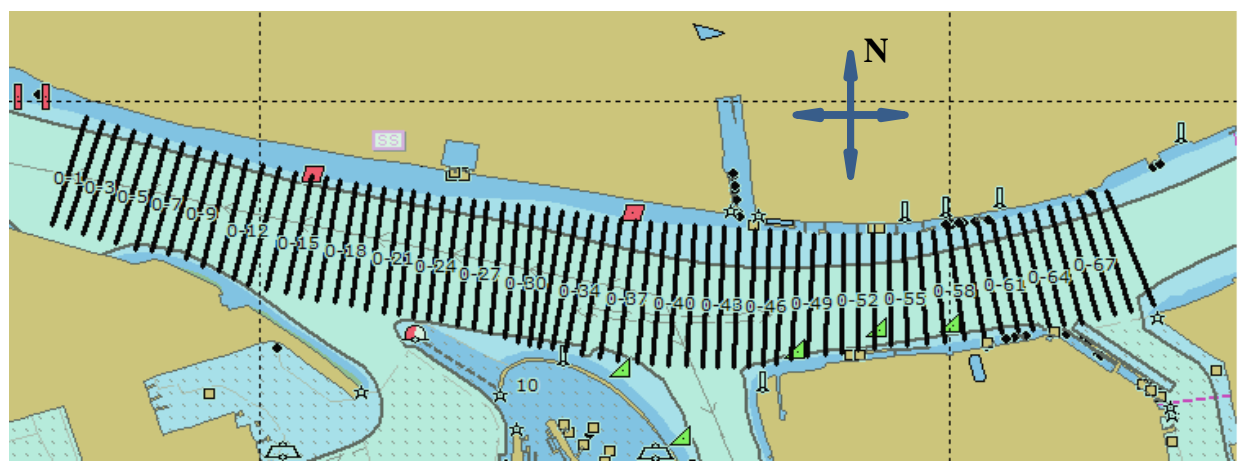

FIGURE 3 Waterway of Sea-Nieuwe Maas and 69 cross sections. 
In our previous research (15), AIS data analysis provided insight into vessel behavior. It was found that vessels deviate from their planned path when they encounter other vessels, especially during overtaking.

As mentioned before, vessel route choice is at the tactical level, where the influence of vessel encounters is not considered. To eliminate the influence of vessel encounters, empirical vessel paths are classified into hindered paths and unhindered paths according to the influence of other vessels. Here, a path is defined unhindered if the distance to other vessels is at least $2 \mathrm{~km}$, during the whole trip of the vessel. AIS data of unhindered paths are then used for the calibration.

However, these unhindered paths concentrate in the right part of the waterway. To be able to estimate the influence of the banks, more data are needed to describe the vessel route choice in the areas close to banks. To provide more data in these areas, parts of hindered vessel paths are used. For hindered vessel paths, vessels normally deviate from their planned path and sail into the area closer to the banks. It is assumed that the influence of other vessels ends after the encounter. At that moment, both vessels have the largest deviation when they are closest to each other. Hindered vessel paths after the encounter can then be considered as unhindered and used for calibration as well. Including these, the tracks of the AIS dataset used for calibration cover most of the waterway, see FIGURE 4. In the figure, longitude and latitude coordinates are transformed into coordinates of the 'Rijksdriehoeksstelsel' (RD), which is the national grid of the Netherlands. This national grid is used as a basis for geographical indications and files, such as Geographic Information Systems.

The definitions of the parameters of the bend waterway are given in FIGURE 1. According to bend strength $\theta / S$, the waterway is divided into two parts: the area from cross section 1 to cross section 42 and the area from cross section 42 to cross section 69. Based on different bend strength, vessels in these two parts will have different contributions to cost from the bend effect.

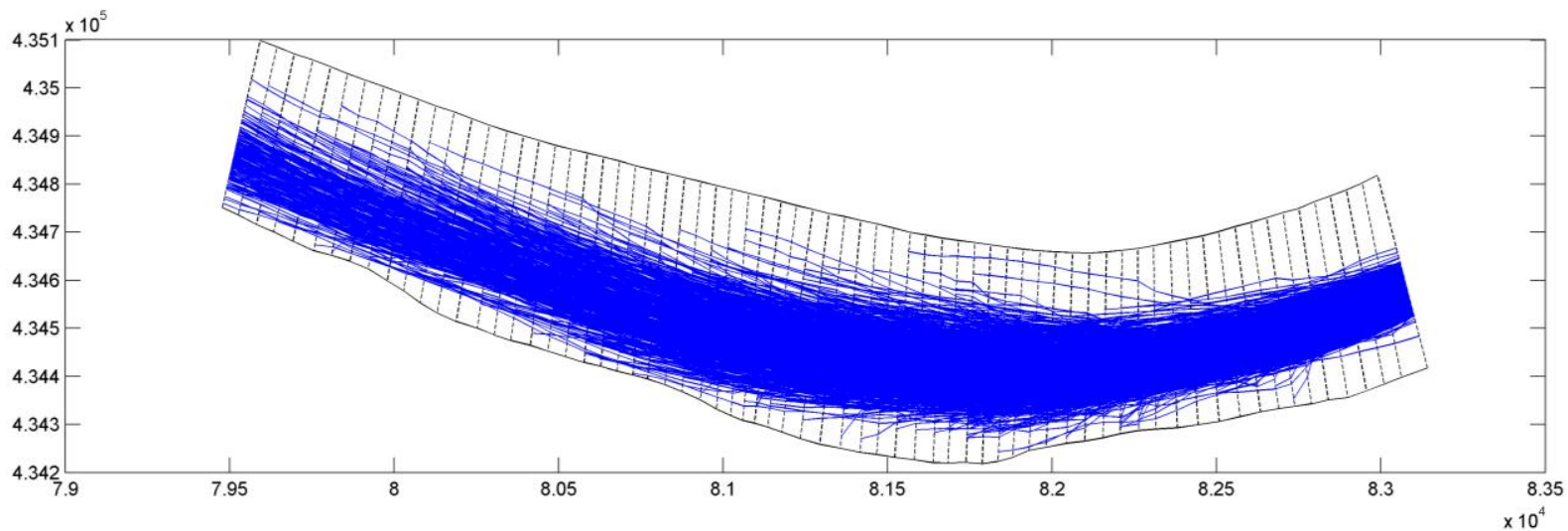

FIGURE 4 AIS data used for vessel route choice calibration from Sea to Nieuwe Maas.

\section{Calibration set-up and objective function}

As mentioned before, only the ratio between the weights can be determined by AIS data. Without loss of generality, we can set $c_{1}=1$. Then, the parameters that need to be calibrated are in the vector $\beta^{T}=\left(c_{2}, c_{3}, c_{4}, r_{1}, r_{2}, c_{5}\right)$.

In our research, vessels have a two-dimensional motion including vessel speed and course. As we discussed before, vessel speed is determined at the operational level by the bridge team according to the vessel engine power or influence of other vessels and external influences (such as wind and visibility), but not at the tactical level. Therefore, only vessel course is considered in the objective function in calibration of route choice model. The calibration process aims at minimizing the difference between vessel course measured from AIS data and vessel course predicted by the vessel route choice model.

As we can see in FIGURE 4, vessel paths concentrate in the right part of the waterway and they are not uniformly distributed. Overlapping paths provide similar inputs to the calibration. To combine a lot of repetitive inputs, a "meshgrid" of $10 \mathrm{~m} \times 10 \mathrm{~m}$ is used to generate velocity field, which will be used to determine the difference. FIGURE 5 shows the generated a velocity field based on the "meshgrid" and AIS data in FIGURE 4. This velocity field will be used to compare with the simulated results based on route choice model. 


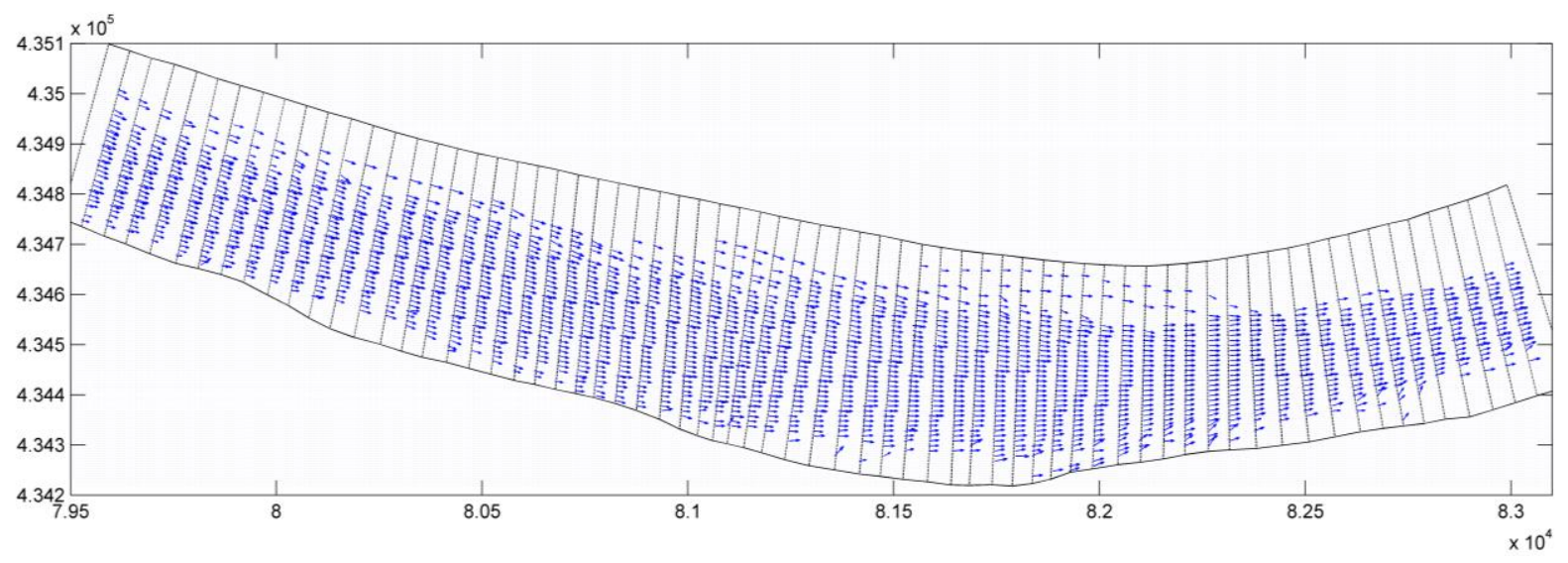

FIGURE 5 Velocity field based on AIS data in the meshgrid of $10 \mathrm{~m} \times 10 \mathrm{~m}$.

In the simulation model, the part of the cross section 69 (the rightmost), where $99 \%$ of the unhindered vessels pass, is defined as the destination. To use the numerical solution approach, we discretize the waterway into small $5 m \times 5 m$-cells and define the time step as 0.5 seconds. Then, the value function can be solved for the whole research area, as well as the optimal course field. As we discussed before, only vessel course is considered in the route choice model calibration. This optimal course field will be used to compare with the velocity field in FIGURE 5 .

Let $\alpha_{\text {data }}$ denote vessel course in the velocity field in FIGURE 5. Correspondingly, $\alpha_{\text {sim }}$ is the optimized course for the same point in the "meshgrid" calculated by the route choice model based on a given $\beta$. For these $m$ "meshgrid" points, the average square error is defined as

$$
E(\beta)=\frac{1}{m} \sum_{i=1}^{m}\left(\alpha_{d a t a}-\alpha_{\text {sim }}\right)^{2}
$$

So the calibration problem becomes a multi-variable nonlinear optimization problem as following, which could be solved by the function "fminsearch" in MATLAB.

$$
\beta^{*}=\arg \min E(\beta)
$$

\section{Calibration Results}

By applying the described optimization method, the best fit of the route choice model to the AIS data is found. The calibration results are summarized in TABLE 1.

TABLE 1 Calibration Results for Vessel Route Choice Model

\begin{tabular}{|c|c|c|c|c|c|c|}
\hline \multirow{2}{*}{$\begin{array}{c}\text { Optimized } \\
\text { parameters }\end{array}$} & $c_{2}$ & $c_{3}$ & $c_{4}$ & $r_{1}$ & $r_{2}$ & $c_{5}$ \\
\cline { 2 - 7 } & 0.0211 & 0.0122 & 0.0215 & 0.5414 & 0.2305 & 0.0218 \\
\hline Error & \multicolumn{7}{|c|}{19.92} \\
\hline
\end{tabular}

In the model, the values of parameters $c_{3}$ and $c_{4}$ reflect the cost when the vessel is very close to the portside bank and starboard bank. $c_{3}$ and $c_{4}$ also have the largest influence from both banks, since the influence of banks is a linear decreasing function of the distance to the bank in equations (9) and (10). That means that banks contribute to the cost function between $0 \sim 0.02$. Compared to the contribution of sailing time (equals 1 ), these two values seem small, but they cannot be neglected as they provide the repellence of both banks.

The parameters $r_{1}$ and $r_{2}$ describe the percentage of the waterway width, in which both banks contribute to the cost function. Calibration results show that portside bank have influence when the distance between the vessel and the portside bank is less than 54\% of the waterway width. For the starboard bank, the influence area is $23 \%$ of the waterway width. The rest area around $23 \%$ of the waterway is the area where banks do not have influence on vessel behavior. This area could be considered as unhindered area, where the vessels will concentrate. This is corresponding to the phenomenon observed in FIGURE 4.

Applying the calibrated results shown in TABLE 1, the expected minimum disutility function is generated in the research area. The contour lines and corresponding value for this value function are shown in FIGURE 6. 
Vessels will follow the preferred route, which is perpendicular to these contour lines. The shape of contour lines indicates that vessels will be pushed away from the bank when they are too close to the bank.

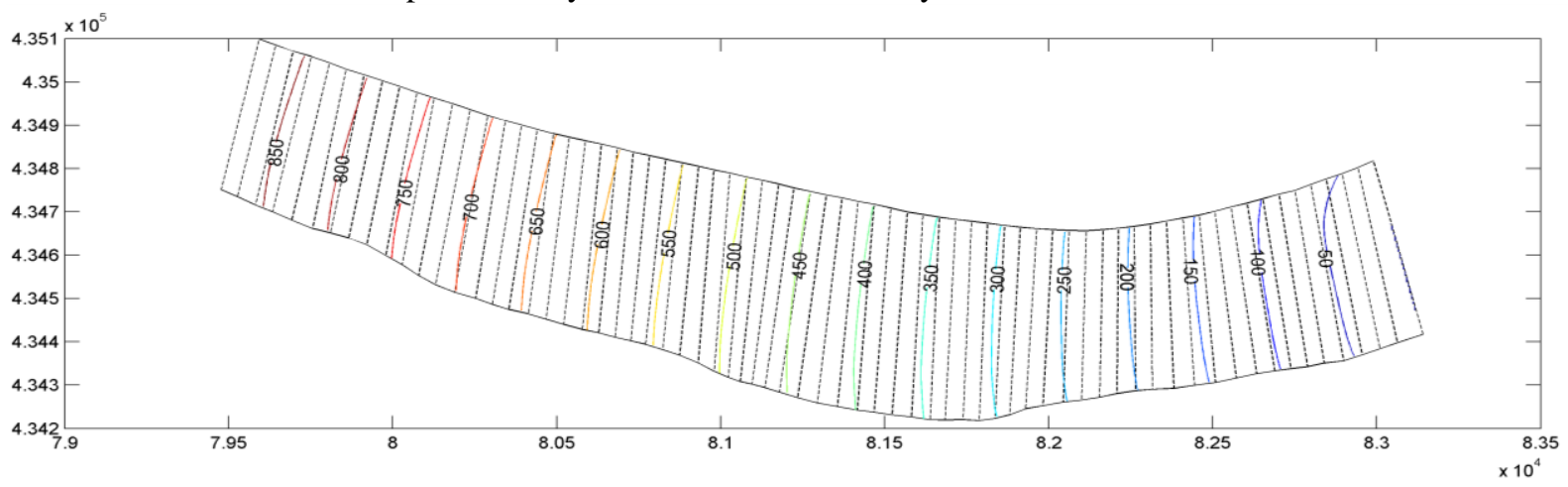

FIGURE 6 Contour lines for value function.

On cross section 2, 20, 40 and 60, several example tracks of route choice are generated in FIGURE 7. It can be seen that when vessels are too close to the bank, they will be pushed away from the bank and sail towards the unhindered area, which is corresponding to the phenomenon in FIGURE 4 and is what we expect.

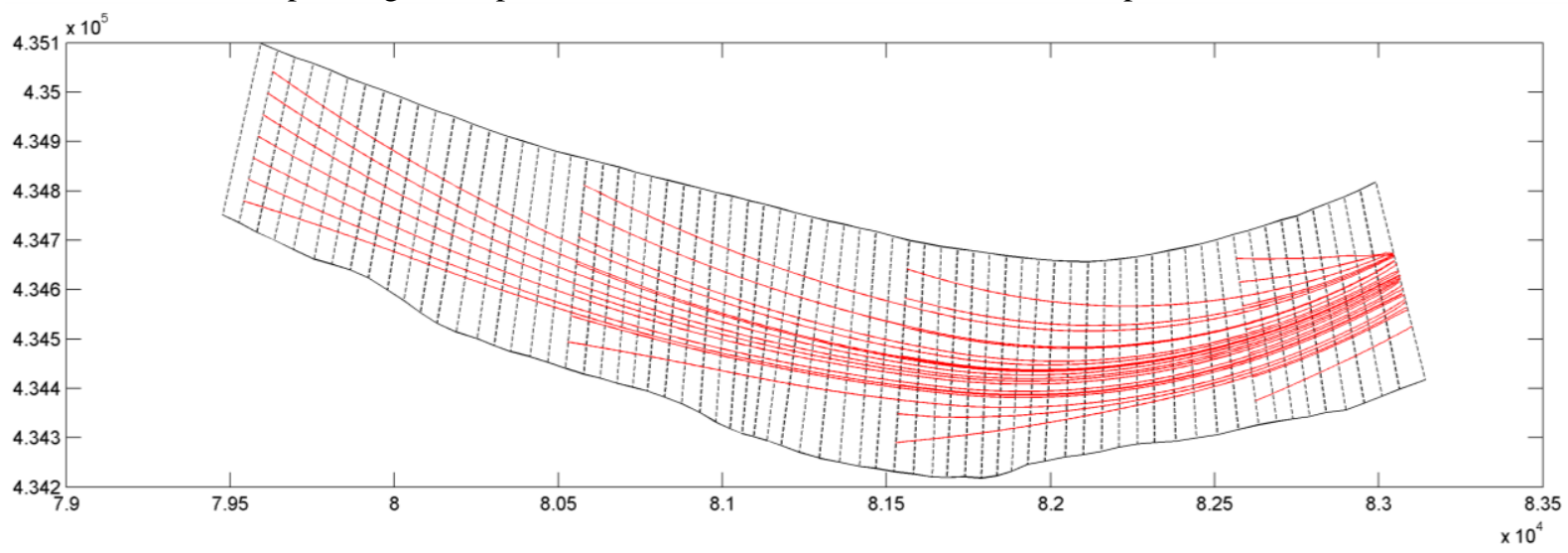

FIGURE 7 Example tracks on cross sections 2, 20, 40 and 60.

\section{CONCLUSIONS AND RECOMMENDATIONS}

In this paper, an approach is proposed to generate vessel route choice in continuous time and space for ports and inland waterways. A dynamic programming approach and a numerical solution approach are used to solve the value function, which can be used to generate optimal course and speed. Furthermore, this vessel route choice model is calibrated based on AIS data of unhindered paths and hindered path.

The calibrated results show the repulsion from two banks, which is corresponding to the observation in AIS data. The repulsion range of the portside bank is around 54\% and the repulsion range of the starboard bank approximately equals to $23 \%$, which result in the fact that vessels concentrate in the right part of the waterway. The results also show plausible example preferred routes in the research area, which help us to understand the desired vessel behavior (route). In the model, the waterway geometry is input, so the route choice model is also applicable in other parts.

The vessel route choice model provides the preferred routes for vessels, which could be used as a reference guide for both the bridge team and Vessel Traffic Services (VTS), and therefore can help to improve the waterway traffic management. In addition, the preferred routes generate by the route choice model could be used to indicate the dangerous area for vessels, considering the maneuverability of vessels. Thus, in decision-making level, the model is able to be used to control the vessel traffic, design new ports or extend existing ports and inland waterways.

Suggestions for future research are the inclusion of cost of other infrastructural elements in the route choice, such as dams and jetties. This way, the actual sailing environment will be reflected in the model and make the model generic. In addition, AIS data sets from other areas and in other sailing directions will be used to calibrate and validate the model. 
Furthermore, the vessel route choice model will serve as input to the maneuvering model at the operational level. Both route choice model and maneuvering model form the new maritime traffic model, which describes maritime traffic by predicting single vessel behavior.

\section{ACKNOWLEDGEMENT}

This research is part of the research program "Nautical traffic model based design and assessment of safe and efficient ports and waterways", sponsored by the Netherlands Organization for Scientific Research (NWO). The authors like to thank MARIN and the Port of Rotterdam for providing AIS data. The fellowship of Yaqing Shu at Delft University of Technology is supported by the Chinese Scholarship Council (CSC).

\section{REFERENCES}

(1) Pedersen, P.T. Collision and grounding mechanics. In WEMT, Copenhagen, 1995.

(2) Fowler, T.G. and E. Sørgård. Modeling ship transportation risk. Risk Analysis, Vol. 20, No. 2, 2000, pp. $225-244$.

(3) Degre, T., C. Glansdorp, and C. van der Tak. The importance of a risk based index for vessels to enhance maritime safety. In the 10th IFAC Symposium on Control in Transportation Systems, Tokyo, Japan, 2003.

(4) Sutulo, S., L. Moreira, and C. Guedes Soares. Mathematical models for ship path prediction in manoeuvring simulation systems. Ocean engineering, Vol. 29, No. 1, 2002, pp. 1-19.

(5) Sariöz, K. and E. Narli. Assessment of manoeuvring performance of large tankers in restricted waterways: a realtime simulation approach. Ocean engineering, Vol. 30, No. 12, 2003, pp. 1535-1551.

(6) Yoon, H.K. and K.P. Rhee. Identification of hydrodynamic coefficients in ship maneuvering equations of motion by Estimation-Before-Modeling technique. Ocean Engineering, Vol. 30, No. 18, 2003, pp. 2379-2404.

(7) Hsu, C.-I. and Y.-P. Hsieh. Routing, ship size, and sailing frequency decision-making for a maritime hub-andspoke container network. Mathematical and Computer Modelling, Vol. 45, No. 7, 2007, pp. 899-916.

(8) Norstad, I., K. Fagerholt, and G. Laporte. Tramp ship routing and scheduling with speed optimization. Transportation Research Part C: Emerging Technologies, Vol. 19, No. 5, 2011, pp. 853-865.

(9) Kosmas, O. and D. Vlachos. Simulated annealing for optimal ship routing. Computers \& Operations Research, Vol. 39, No. 3, 2012, pp. 576-581.

(10) Hoogendoorn, S.P. Normative Pedestrian Flow Behavior, Theory and Applications. Delft University of Technology, Faculty of Civil Engineering and Geosciences, Transportation and Traffic Engineering section, Delft, the Netherlands, 2001.

(11) Hoogendoorn, S., W. Daamen, Y. Shu, and H. Ligteringen. Modeling human behavior in vessel maneuver simulation by optimal control and game theory. In Transportation Research Record: Journal of the Transportation Research Board, No. 2326, Transportation Research Board of the National Academies, Washington, D.C., 2013, pp. 45-53.

(12) Gunnar Aarsæther, K. and T. Moan. Estimating navigation patterns from AIS. Journal of Navigation, Vol. 62, No. 04, 2009, pp. 587-607.

(13) Mou, J.M., C.v.d. Tak, and H. Ligteringen. Study on collision avoidance in busy waterways by using AIS data. Ocean Engineering, Vol. 37, No. 5, 2010, pp. 483-490.

(14) Shu, Y., W. Daamen, H. Ligteringen, and S. Hoogendoorn. Vessel Speed, Course, and Path Analysis in the Botlek Area of the Port of Rotterdam, Netherlands. In Transportation Research Record: Journal of the Transportation Research Board, No. 2330, Transportation Research Board of the National Academies, Washington, D.C., 2013, pp. 63-72.

(15) Shu, Y., W. Daamen, H. Ligteringen, and S. Hoogendoorn. AIS data analysis for vessel behavior during strong currents and during encounters in the Botlek area in the Port of Rotterdam. In The International Workshop on Nautical Traffic Models, Delft, the Netherlands, 2013.

(16) Fleming, W.H., H.M. Soner, and H.M. Soner. Controlled Markov processes and viscosity solutions. Springer, 2006.

(17) Hoogendoorn, S.P. and P.H. Bovy. Pedestrian route-choice and activity scheduling theory and models. Transportation Research Part B: Methodological, Vol. 38, No. 2, 2004, pp. 169-190. 